\title{
Analysis of the Risk Factors and their Association with Diabetes among Population of African-Americans
}

Mehdi Rahimi and Minggen Lu

University of Nevada-Reno ; Email: mrahimi@unr.edu 


\section{Introduction}

\section{Prevalence}

Over 415 million people live with diabetes worldwide, and an estimated 193 million people have undiagnosed diabetes. Type 2 diabetes accounts for more than $90 \%$ of patients with diabetes and leads to microvascular and macrovascular complications that cause profound psychological and physical distress to both patients and care givers and put a huge burden on health-care systems (Chatterjee, Khunti \& Davies, 2017, p. 2239).

In the United States, an estimated 30.3 million persons have diabetes based on the data from 2015 and only 23.1 million of these cases have been diagnosed out of which $12.7 \%$ were African American (American Diabetes Association, Diabetes Statistics, 2015). The incidence of diabetes is increasing because of the aging and changing ethnic mix of the population and because of worsening obesity. On the basis of current trends, the prevalence of diabetes is expected to nearly double by 2030 (Wild, Roglic, Green, Sicree, \& King, 2004).

\section{Causes and Complications}

Type 2 diabetes, once known as adult-onset or noninsulin-dependent diabetes, is a chronic condition that affects the way body metabolizes sugar. Type 2 diabetes is characterized by impaired insulin secretion. Some but not all studies suggest that a decrease in $\beta$-cell mass contributes to this (Butler, Janson, BonnerWeir, Ritzel, Rizza, \& Butler, 2003).

Diabetes can cause serious health problems, such as heart disease, stroke, and eye and foot problems. Prediabetes also can cause health problems. Cardi-cerebrovascular disease are frequently associated to type 2 diabetes and may become life threatening, particularly coronaropathy, stroke and heart failure (Schlienger, (2013). Diabetic foot is a severe complication secondary to microangiopathy, 
microangiopathy and neuropathy. It may be considered as a super-complication of several complications. Some cancer may be considered as an emerging complication of type 2 diabetes as well as cognitive decline, sleep apnea syndrome, mood disorders and bone metabolism impairments (Schlienger, (2013).

\section{Signs and Symptoms}

Signs and symptoms of the type 2 diabetes often develop slowly. Some of these signs and symptoms include: 1) Increased thirst and frequent urination. 2) Increased hunger. 3) Weight loss. 4) Fatigue. 5) Blurred vision. 6) Slow-healing sores or frequent infections. 7) Areas of darkened skin. This condition, called Acanthosis Nigerians, may be a sign of insulin resistance (Mayo Foundation for Medical Education and Research, Type 2 diabetes, 2015).

\section{Risk Factors}

The chances of developing type 2 diabetes depend on a combination of risk factors. It's clear, however, that certain factors increase the risk more than others.

Physical activity and diet can have a direct effect on the obesity which is a key risk factor for diabetes (Mokdad, Bowman, Ford, Vinicor, Marks, \& Koplan, 2001). A 7-year prospective study showed that the waist circumference is the best predictor of noninsulin dependent diabetes mellitus (NIDDM) compared to body mass index, waist/hip ratio and other anthropometric measurements (Wei, Gaskill, Haffner, \& Stern, 1997).

Race is also an important factor. African American, Hispanic/Latino American, American Indian, Alaska Native, Native Hawaiian, or Pacific Islander have a higher risk factor (The National Institute of Diabetes and Digestive and Kidney Diseases Health Information Center, Risk Factors for Type 2 Diabetes, 2016).

Age is another important risk factor. Therefore it has been suggested that screening to be done for diabetes every 3 years in persons older than 45 years and in persons younger than 45 years (American 
Diabetes Association, 2010). According to Chung et al (2014), universal screening of individuals aged more than or equal to 35 years would simplify the current complex criteria for assessment for screening eligibility in primary care.

Family history of type II diabetes also appears to increase the risk of type II diabetes and of coronary heart disease. Both leptin and adiponectin show a significant association with family history of diabetes, independently of age, body mass index, insulin resistance, glucose, lipids, and blood pressure levels (De Pergola, Manicone, Lovero, Simone, Bruno, \& Zamboni, 2008).

As an overall view to the risk factors, we can considers the categorization of some organizations. The NIH has determined these factors: 1) Overweight, 2) Age 45 or older, 3) Family history, 4) Race, 5) Not physically active, 6) High blood pressure, 7) Low HDL or high triglycerides, 8) History of heart disease, 9)Depression (The National Institute of Diabetes and Digestive and Kidney Diseases Health Information Center, 2016).

The CDC also has identified similar risk factor such as these: 1) Overweight, 2) Age 45 years or older

Family history, 3) Race, 4) Not physically active, 5) History of gestational diabetes, 6) History of polycystic ovary syndrome (National Center for Chronic Disease Prevention and Health Promotion, 2017).

Other organizations have identified similar risk factor which are included in the appendix. Based on these comparison, we can conclude that weight, age, family history, race and physical activity are the most important risk factors.

\section{Prevalence among African-Americans}

Type 2 diabetes is an important problem among African-Americans. In the USA, compared to whites, African-Americans are disproportionately impacted by the diabetes epidemic (Chatterjee, Maruthur, \& Edelman, 2015). Diabetes is over twice as prevalent among African-Americans as compared to whites 
(Brancati, Whelton, Kuller, \& Klag, 1996). Traditional diabetes risk factors, such as obesity, are more common among African-Americans. (Chatterjee, et al, 2015). The excess prevalence of diabetes among African- Americans contributes to their disturbingly high rates of diabetic end-stage renal disease and lower-extremity amputation (Tunis, Bass, Klag, \& Steinberg, 1993). While African-Americans have a higher prevalence of some of the more common diabetes risk factors, particularly obesity and lower socioeconomic status, these traditional risk factors do not explain all of the racial disparity of diabetes risk (Chatterjee, et al, 2015). It has been suggested that an additional $30 \%$ of the racial disparity in diabetes risk arises from risk factors not yet accounted for (Chatterjee, et al, 2014).

\section{Purpose}

The purpose of this project is to determine the pattern and risk factors for diabetes among a population of African-American served by University of Virginia School of Medicine.

\section{Methods}

In this project, we will analyze a dataset that consist of 13 variables on 403 subjects from 1046 subjects. The goal of the study is to determine whether these variables were risk factors in clinic population being served by University of Virginia School of Medicine. These subjects were interviewed in a study at University of Virginia School of Medicine to understand the prevalence of obesity, diabetes, and other cardiovascular risk factors in central Virginia for African Americans. The 403 subjects were the ones who were actually screened for diabetes.

The variable "Disease" is defined as when the Glycosolated hemoglobin is more than 7.0 as a positive diagnosis of diabetes. The BMI is also calculated using the weight and height and a new categorical factor 
called "obesity" was defined based on the BMI with three levels (Underweight and normal, Overweight, Obese). The "Underweight and Normal" level was selected as the reference.

Another ratio is defined too as the ratio of the hip measurement to the waist measurement. This was calculated to consider the possibility that this ratio may explain obesity better than $\mathrm{BMI}$ alone considering the shortcoming of the BMI.

Percentage, frequency of distribution, mean, and standard deviation were used to describe the characteristics of variables. The test of normality was performed on each data variable with the null hypothesis that the outcome is normally distributed. The emphasis was put on the Kolmogorov-Smirnov test but the other tests were considered too. The histogram and the boxplot of each of the variables is provided in the appendix. The odds ratios were calculated too and are reported in the tables.

Chi-square tests were performed on the categorical variables to test the difference of proportions.

Univariate analysis was used on all the variables to identify the potential factors that were associated with the diabetes.

At the end, a regression model for the association between the risk factors and the disease was obtained. The Likelihood Ratio statistic was used to test specific risk factors in the model. Logistic regression model was used to achieve this model. The variable "age" was held as the biological factor in this part.

The data were analyzed with SAS (Statistical Analysis System) version 9.4 for Windows (SAS Institute, Cary, NC, USA).

In all of the tests, values of $p<0.05$ were considered as statistically significant. 


\section{Results}

Summary statistics of this dataset are provided in Table 1 and Table 2 for the categorical and continuous variables.

In regards to the gender of the subjects, $58 \%$ were female and the rest are male. (The "female" level was selected as the reference). There is no missing data point in this regard. As for the indication of the disease, about $15 \%$ were identified as having diabetes. Out of the 403 subjects, 13 had missing data points for indicating the disease and will not be used in the study.

A new categorical value called "Obesity" was defined based on the BMI on three levels of Underweight or Normal, Overweight and Obese. This was done using dummy variables. Further explanation of this can be found in the appendix. This variable had 6 missing data points.

A continuous variable called "ratiohw" was defined as the ration of the hip measurement to the waist measurement. This was introduced as another approach for measuring the obesity rather than using BMI.

Table 1. Summary of the categorical variables in the study

\begin{tabular}{llrr}
\hline Variable & Levels & $\mathbf{N}$ & Percent \\
\hline Gender & Male & 169 & $41.94 \%$ \\
& Female & 234 & $58.06 \%$ \\
\hline Disease & 1 = yes & 60 & $15.38 \%$ \\
\hline Obesity & $1=$ no & 330 & $84.62 \%$ \\
& $1=$ Underweight or Normal & 119 & $29.97 \%$ \\
& $2=$ Overweight & 125 & $31.49 \%$ \\
\hline
\end{tabular}


Table 2. Summary of the continuous variables in the study

\begin{tabular}{|c|c|c|c|c|c|c|}
\hline Variable & $\mathbf{N}$ & Mean & Median & Std. Dev. & Minimum & Maximum \\
\hline chol & 402 & 207.85 & 204 & 44.45 & 78 & 443 \\
\hline hdl & 402 & 50.45 & 46 & 17.26 & 12 & 120 \\
\hline ratio & 402 & 4.52 & 4.2 & 1.73 & 1.5 & 19.3 \\
\hline glyhb & 390 & 5.59 & 4.84 & 2.24 & 2.68 & 16.11 \\
\hline age & 403 & 46.85 & 45 & 16.31 & 19 & 92 \\
\hline height & 398 & 66.02 & 66 & 3.92 & 52 & 76 \\
\hline weight & 402 & 177.59 & 172.5 & 40.34 & 99 & 325 \\
\hline sbp & 398 & 136.9 & 136 & 22.74 & 90 & 250 \\
\hline dbp & 398 & 83.32 & 82 & 13.59 & 48 & 124 \\
\hline waist & 401 & 37.9 & 37 & 5.73 & 26 & 56 \\
\hline hip & 401 & 43.04 & 42 & 5.66 & 30 & 64 \\
\hline BMI & 397 & 28.85 & 27.86 & 6.62 & 15.24 & 55.9 \\
\hline ratiohw & 401 & 1.143 & 1.136 & 0.093 & 0.875 & 1.466 \\
\hline
\end{tabular}

As it can be seen in Table 2, there are some missing values in this study that need to be accounted for. The histogram and the boxplot of each of the variables is provided in the appendix. It was also noticed that other than "height" and "age", all other variables had outliers on the upper bound and almost no outliers on the lower bound.

It should be noted that the "ratiohw" variable is the ratio of hip to waist and therefore the smaller values could be considered as an indicator of obesity. 


\section{Chi-square Test of Association}

The chi-square test was used to see the association between each variable and the disease and to give us a bigger picture of the variable selection for finding the association. Each variable was categorized into four groups: 1) Minimum value to the $25^{\text {th }}$ percentile, 2) $25^{\text {th }}$ percentile to the median, 3) Median to the $75^{\text {th }}$ percentile, 4) $75^{\text {th }}$ percentile to the maximum.

The null hypothesis was that there is no association and the alternative hypothesis was that there is an association between the variable and the disease.

The Chi-square p-value for the "waist", "hip", "BMI", "age", "sbp" and "chol" were less than 0.05 and could be considered as potentially having association with disease.

The p-values of "weight", "height", "dbp", "hdl" and "gender" were above 0.05 and therefore were considered as not having an association with the disease by the test of Chi-square.

This test was used only for giving a bigger picture of the association of the variables and the univariate analysis was used a better tool.

\section{Univariate Analysis}

In this section, we present the estimated effect for each variable using univariate analysis. The cutoff value for each variable to be included in the Likelihood test was set at 0.05 but values more than that and below 0.2 could be considered too. In this dataset, such a situation did not happen and variables of "height", "dbp" and "gender" were all above 0.2 and therefore were not included in the likelihood ratio test.

The rest of the variables were considered as a potential risk factors and were included in the model selection. The variable of "age" was selected as a biological variable based on the literature review presented in the Introduction section. 
Table 3. Estimated effect for each variable based on separate univariate logistic regression models

\begin{tabular}{|c|c|c|c|c|c|}
\hline Variable & Estimate & SE & OR & $95 \% \mathrm{Cl}$ & p-value \\
\hline chol & 0.0115 & 0.0030 & 1.0116 & $(1.006,1.018)$ & 0.0001 \\
\hline hdl & -0.0237 & 0.0098 & 0.9766 & $(0.958,0.996)$ & 0.0161 \\
\hline ratio & 0.3993 & 0.0864 & 1.4908 & $(1.259,1.766)$ & $<.0001$ \\
\hline age & 0.0525 & 0.0094 & 1.0539 & $(1.035,1.073)$ & $<.0001$ \\
\hline height & 0.0142 & 0.0361 & 1.0143 & $(0.945,1.089)$ & 0.6936 \\
\hline weight & 0.0103 & 0.0033 & 1.0104 & $(1.004,1.017)$ & 0.0019 \\
\hline sbp & 0.0212 & 0.0057 & 1.0214 & $(1.010,1.033)$ & 0.0002 \\
\hline dbp & 0.0100 & 0.0103 & 1.0100 & $(0.990,1.031)$ & 0.3316 \\
\hline waist & 0.1032 & 0.0244 & 1.1087 & $(1.057,1.163)$ & $<.0001$ \\
\hline hip & 0.0644 & 0.0235 & 1.0665 & $(1.019,1.117)$ & 0.0061 \\
\hline BMI & 0.0547 & 0.0202 & 1.0562 & $(1.015,1.099)$ & 0.0067 \\
\hline Gender & 0.0869 & 0.2835 & 1.0480 & $(1.018,1.078)$ & 0.7591 \\
\hline ratiohw & -5.4454 & 1.6110 & 0.0043 & $(0.0002,0.1015)$ & 0.0007 \\
\hline Obesity (Overall) & & & & & 0.0188 \\
\hline Overweight vs. Normal & 0.8468 & 0.4248 & 2.3322 & $(1.014,5.362)$ & \\
\hline Obesity vs. Normal & 1.0544 & 0.4043 & 2.8702 & $(1.299,6.339)$ & \\
\hline
\end{tabular}

\section{Variable Selection}

The goal of this section is to build a model to identify a set of variables that offer a satisfactory explanation of the disease occurrence in the study population. 
Since the "ratio" variable is the ratio of "chol" to "hdl", only the "ratio" was included in the model selection and not those two variables. The same was decided for the "ratiohw" where "hip" and "waist" were not included in the model selection. Similarly, the same logic was applied to BMI and "height" and "weight".

Table 4. Likelihood Ratio tests for diabetes risk factors. ("age" is a biological factor)

\begin{tabular}{|c|c|c|c|c|c|}
\hline Model & Terms & Log-Likelihood & Chi-Square & df & p-value \\
\hline \multirow[t]{5}{*}{1} & $\mathrm{int}+$ age + ratio $+\mathrm{sbp}+$ ratiohw + bmi2 + bmi3 & -131.3327 & - & - & - \\
\hline & - bmi2 - bmi3 & -137.6555 & 12.6456 & 2 & 0.0018 \\
\hline & - ratiohw & -132.1470 & 1.6286 & 1 & $\underline{0.2019}$ \\
\hline & $-s b p$ & -132.2731 & 1.8808 & 1 & 0.1702 \\
\hline & - ratio & -139.7187 & 16.772 & 1 & $<0.0001$ \\
\hline \multirow[t]{4}{*}{2} & int + age + ratio + sbp + bmi $2+b m i 3$ & -132.1470 & - & - & - \\
\hline & - bmi2 - bmi3 & -138.2481 & 12.2022 & 2 & 0.0022 \\
\hline & $-s b p$ & -133.0572 & 1.8204 & 1 & $\underline{0.1773}$ \\
\hline & - ratio & -140.7680 & 17.242 & 1 & $<0.0001$ \\
\hline \multirow[t]{3}{*}{3} & int + age + ratio + bmi $2+$ bmi 3 & -133.0572 & - & - & - \\
\hline & - bmi2 - bmi3 & -139.3227 & 12.531 & 2 & 0.0019 \\
\hline & - ratio & -141.8020 & 17.4896 & 1 & $<0.0001$ \\
\hline
\end{tabular}

As given in Table 4, the Likelihoods Ratio was used to test a specific risk factor in the model by comparing the full model containing the risk factors to a reduced model without the factor. Using this method, it was determined that model number 3 can best describe the association. 


$$
\operatorname{logit}[\pi(x)]=\beta_{0}+\beta_{1} \cdot \text { age }+\beta_{2} \cdot \text { ratio }+\beta_{3} \cdot \text { bmi } 2+\beta_{4} \cdot \text { bmi3 }
$$

Table 5 shows this model and the associated beta values.

Table 5. Diabetes Model 3

\begin{tabular}{llllll}
\hline Variable & Estimate & SE & Chi-Square & df & p-value \\
\hline Intercept & -6.6918 & 0.8222 & 66.25 & 1 & $<.0001$ \\
\hline Age & 0.0529 & 0.0103 & 26.47 & 1 & $<.0001$ \\
\hline Ratio & 0.3498 & 0.0907 & 14.88 & 1 & 0.0001 \\
\hline BMI2 & 0.5274 & 0.4603 & & 2 & 0.0940 \\
\hline BMI3 & 0.7407 & 0.4423 & & & 2.80 \\
\end{tabular}

Therefore, the final proposed model is:

$$
\operatorname{logit}[\pi(x)]=-6.6918+0.0529 \times \text { age }+0.3498 \times \text { ratio }+0.5274 \times \text { bmi } 2+0.7407 \times \text { bmi } 3
$$

\section{Discussion}

In this study, we examined the risk factors and their association with diabetes among a population of African-American served by the University of Virginia School of Medicine. This study used data from 403 subjects which covered 13 variables. New variables were also introduced based on calculations of the provided variables. These included BMI, ratio of total cholesterol to the HDL, ratio of hip measurement to the waist measurement and obesity which was defined based on the BMI. The normality of these variables were tested with the Kolmogorov-Smirnov test and graphical figures represented in the appendix. 
Numerous statistical methods were used to indicate the potential factors. The Chi-square Test of Association was used to give an overall view of the potential factors but the Univariate Analysis was used at the end to select the potential factors. Finally, the likelihood ratio test was used to find the best model to describe the association between the risk factors and the diabetes. As for the likelihood ratio test routine, the backward variable selection was used meaning that each time, the full mode was compared with a reduced model where one factor was removed.

Through the literature review, the parameter of "age" was selected as a biological factor and was not removed during this process of selection of the model.

Since some of the variables such as BMI or the two ratios were calculated based on other variables, those base variables were not included in the selection method. The categorical variable of obesity was also used instead of a continuous variable of BMI.

The goal of this process was obtaining a model of association between the risk factors and the diabetes. It was determined that the age, the ratio of total cholesterol to HDL and obesity were the main risk factors that describe the proposed model.

Since many of the variables were replaced by the ratio variables or more general categorical variables, it can be argued that not using these combinations of variables might lead to slightly different results.

The beta values obtained from the model can also indicate how important each factor is in respect to others. By studying the model, it can be noticed that the factor of obesity has the highest effect in the model. The next important factor is the ratio of the total cholesterol to the HDL. Finally the age was effective too.

These results perfectly reconfirm the previously founded risk factors in other researches. The obesity is indeed the most important factor for the diabetes. The odd ratio of Overweight versus Normal weight 
was 2.3322 which was significant and the odd ratio of Obesity versus Normal weight was 2.8702 which was also significant.

This study was focused only on African-Americans and did not include any other racial groups. The reason was that the diabetes is over twice as prevalent among African-Americans as compared to whites. The prevalence of the diabetes among African-American in this area can be explained based on the obesity factor since this factor is the most important problem among this specific population as mentioned in the Introduction section. This result was confirmed by other studies before.

The most important conclusion of this study in regards to preventing the diabetes can be to control weight and blood cholesterol as getting older since these were the risk factors associated with the diabetes.

Therefore, the clinical relevance of these findings is that more screening needs to be done on the blood cholesterol and the weight of older patients as a method of preventing diabetes.

It should be noted that this is a retrospective study and therefore has several limitations, the most important of which is the lack of important variables that were previously found in other studies to be potential risk factors in regards to the diabetes such as physical activity or family history.

Despite such limitations though, this study does provide important information on the risk factors and the model of association between these risk factors and the diabetes. The model can be used to identify the importance of each factor and also to verify the data.

Further studies can be still done this subject. The effect of physical activity and family history on the diabetes has been known for a long time and need to be considered here too. The Prediabetes and also the fat distribution in the body is also a few of interesting factors that can be considered. This means a broader study that covers more factors can be useful in better recognizing the risk factors for diabetes and a more complete model can be constructed. 


\section{References}

American Diabetes Association. (2010). Standards of Medical Care in Diabetes-2010. Diabetes Care, 33(Suppl 1), S11-S61.

American Diabetes Association. Diabetes Statistics. 2015. Accessed at http://www.diabetes.org/diabetesbasics/statistics/ on 2 May 2018.

Brancati, F. L., Whelton, P. K., Kuller, L. H., \& Klag, M. J. (1996). Diabetes mellitus, race, and socioeconomic status a population-based study. Annals of epidemiology, 6(1), 67-73.

Butler, A. E., Janson, J., Bonner-Weir, S., Ritzel, R., Rizza, R. A., \& Butler, P. C. (2003). $\beta$-cell deficit and increased $\beta$-cell apoptosis in humans with type 2 diabetes. Diabetes, 52(1), 102-110.

Chatterjee, R., Maruthur, N. M., \& Edelman, D. (2015). Novel risk factors for type 2 diabetes in AfricanAmericans. Current diabetes reports, 15(12), 103.

Chatterjee, S., Khunti, K., \& Davies, M. J. (2017). Type 2 diabetes. The Lancet, 389(10085), 2239-2251.

Chatterjee, R., Brancati, F. L., Shafi, T., Edelman, D., Pankow, J. S., Mosley, T. H., \& Yeh, H. C. (2014). Nontraditional risk factors are important contributors to the racial disparity in diabetes risk: the atherosclerosis risk in communities study. Journal of general internal medicine, 29(2), 290-297.

Chung, S., Azar, K. M., Baek, M., Lauderdale, D. S., \& Palaniappan, L. P. (2014). Reconsidering the age thresholds for type II diabetes screening in the US. American journal of preventive medicine, $47(4)$, $375-381$.

De Pergola, G., Manicone, M., Lovero, R., Simone, D., Bruno, G., \& Zamboni, M. (2008). Influence of a family history of type II diabetes on fasting leptin and adiponectin plasma levels. Mediterranean Journal of Nutrition and Metabolism, 1(2), 121-127. 
Mayo Foundation for Medical Education and Research (MFMER), Type 2 diabetes, 2015, Accessed at https://www.mayoclinic.org/diseases-conditions/type-2-diabetes/symptoms-causes/syc20351193 on 2 May 2018.

Mokdad, A. H., Bowman, B. A., Ford, E. S., Vinicor, F., Marks, J. S., \& Koplan, J. P. (2001). The continuing epidemics of obesity and diabetes in the United States. Jama, 286(10), 1195-1200.

National Center for Chronic Disease Prevention and Health Promotion, Type 2 Diabetes, 2017, Accessed at https://www.cdc.gov/diabetes/basics/risk-factors.html on 2 May 2018

Schlienger, J. L. (2013). Type 2 diabetes complications. Presse medicale (Paris, France: 1983), 42(5), 839848.

The National Institute of Diabetes and Digestive and Kidney Diseases Health Information Center (NIDDK), Risk Factors for Type 2 Diabetes, 2016, Accessed at https://www.niddk.nih.gov/healthinformation/diabetes/overview/risk-factors-type-2-diabetes on 2 May 2018.

Tunis, S. R., Bass, E. B., Klag, M. J., \& Steinberg, E. P. (1993). Variation in utilization of procedures for treatment of peripheral arterial disease: a look at patient characteristics. Archives of internal medicine, 153(8), 991-998.

Wei, M., Gaskill, S. P., Haffner, S. M., \& Stern, M. P. (1997). Waist circumference as the best predictor of noninsulin dependent diabetes mellitus (NIDDM) compared to body mass index, waist/hip ratio and other anthropometric measurements in Mexican Americans-a 7-year prospective study. Obesity, $5(1), 16-23$

Wild, S., Roglic, G., Green, A., Sicree, R., \& King, H. (2004). Global prevalence of diabetes: estimates for the year 2000 and projections for 2030. Diabetes care, 27(5), 1047-1053. 


\section{Appendix}

Table appendix-1. Comparison of risk factor of type 2 diabetes among various organizations

\begin{tabular}{lll}
\hline NIH Risk Factors: & CDC Risk Factors: & Mayo Foundation Risk Factors: \\
\hline Overweight & Overweight & Weight \\
Age 45 or older & Age 45 years or older & Age \\
Family history & Family history & Family history \\
Race & Race & Race \\
Not physically active & Not physically active & Physical inactivity \\
High blood pressure & History of gestational diabetes & Fat distribution \\
Low HDL or high triglycerides & Having polycystic ovary & Prediabetes \\
History of heart disease & syndrome & Gestational diabetes \\
Depression & (CDC, Type 2 Diabetes, 2017) & Polycystic ovarian syndrome \\
(NIDDK, Risk Factors for Type 2 & & (MFMER, Type 2 diabetes, 2015) \\
Diabetes, 2016) & & \\
\hline
\end{tabular}



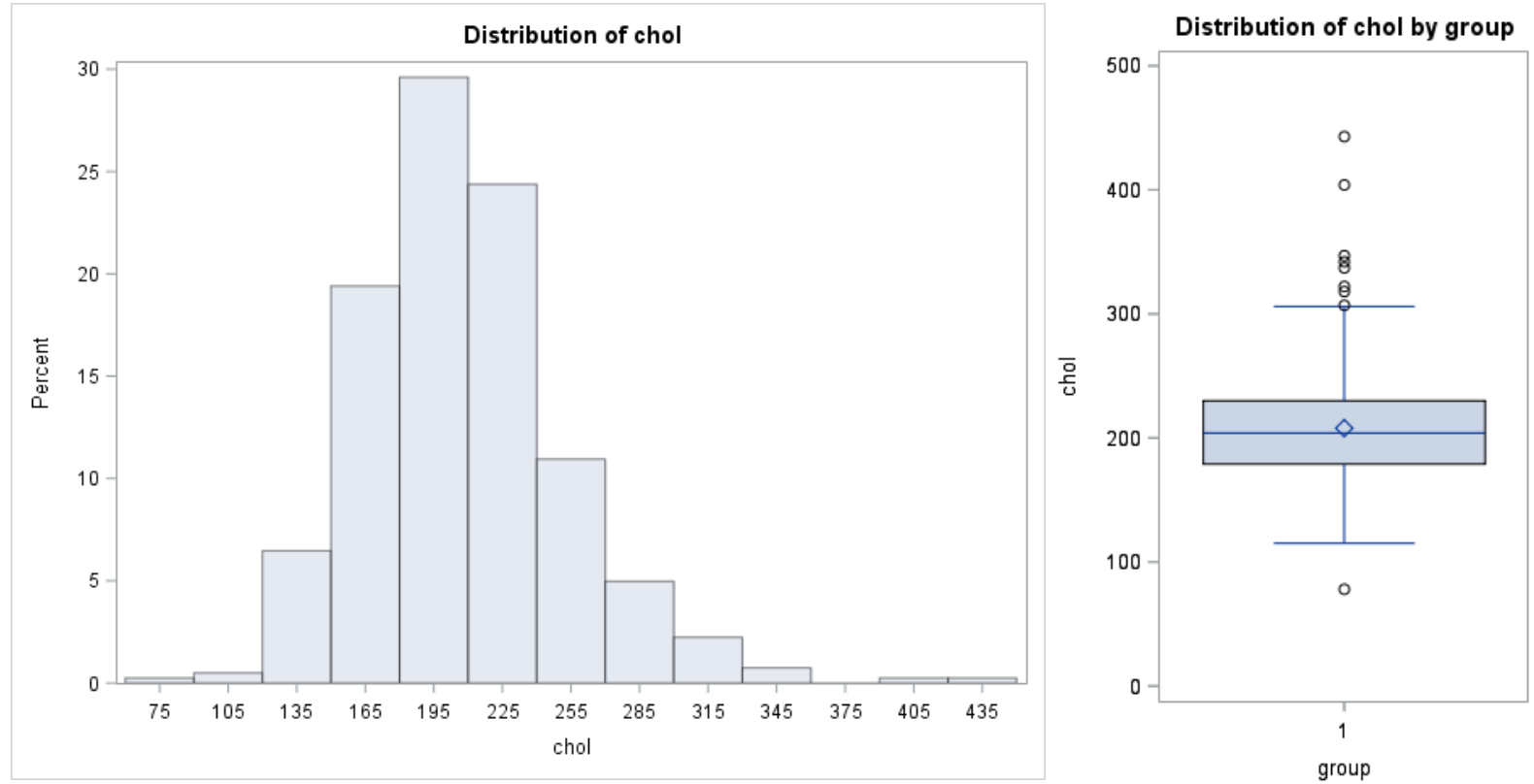

Figure appendix-1. The Histogram and Boxplot of the variable "chol" is shown here.
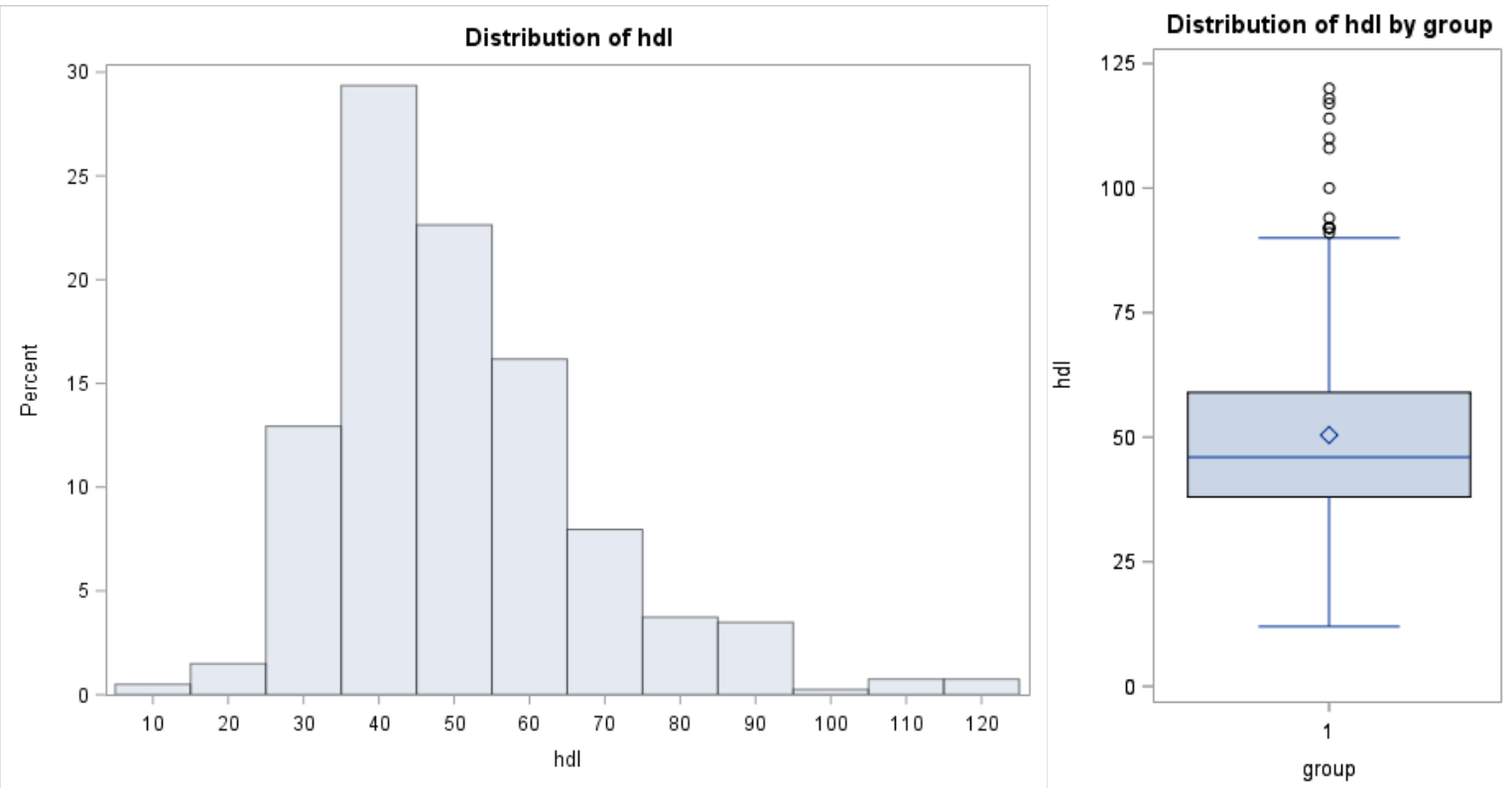

Figure appendix-2. The Histogram and Boxplot of the variable "hdl" is shown here. 

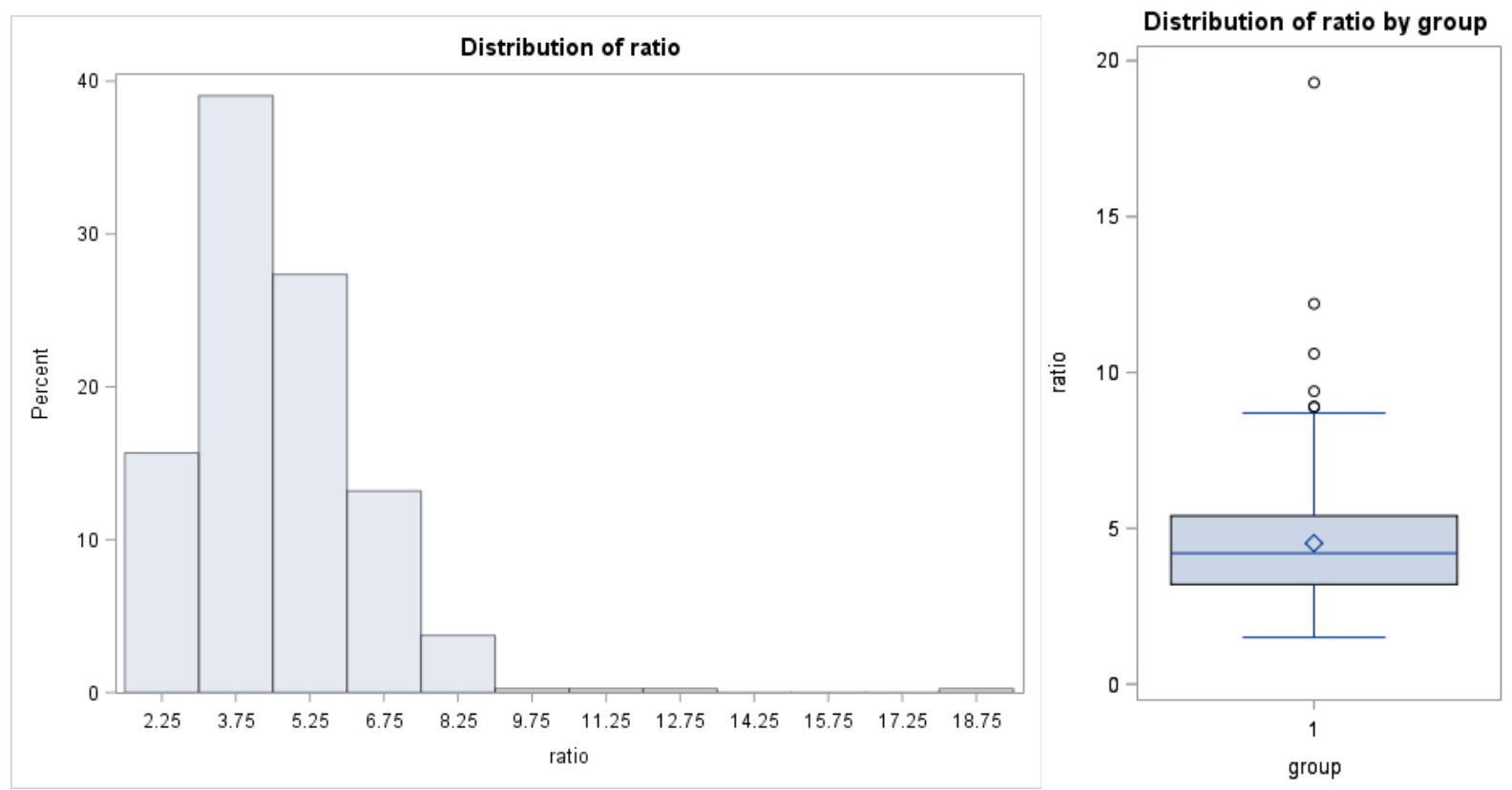

Figure appendix-3. The Histogram and Boxplot of the variable "ratio" is shown here.
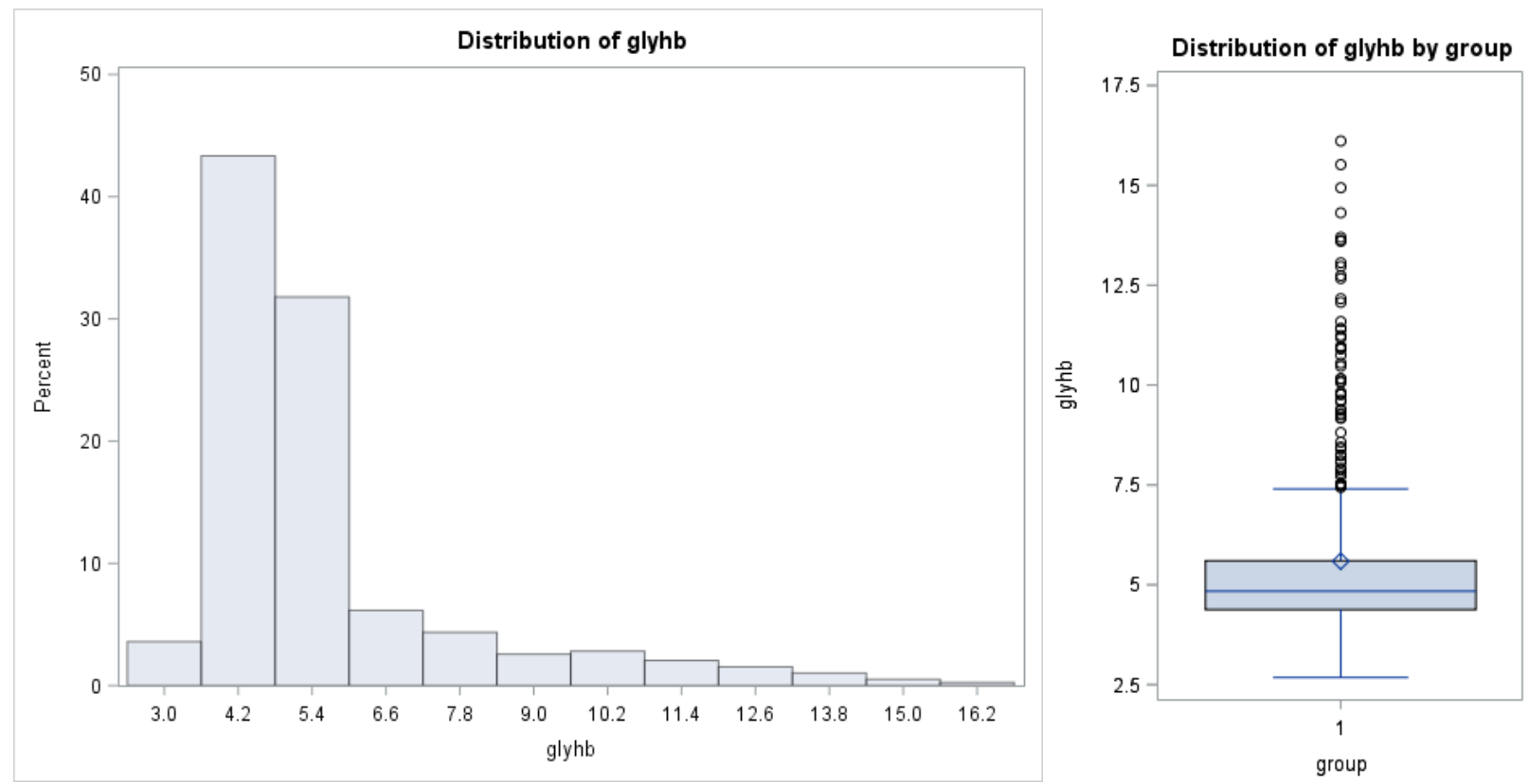

Figure appendix-4. The Histogram and Boxplot of the variable "glyhb" is shown here. 

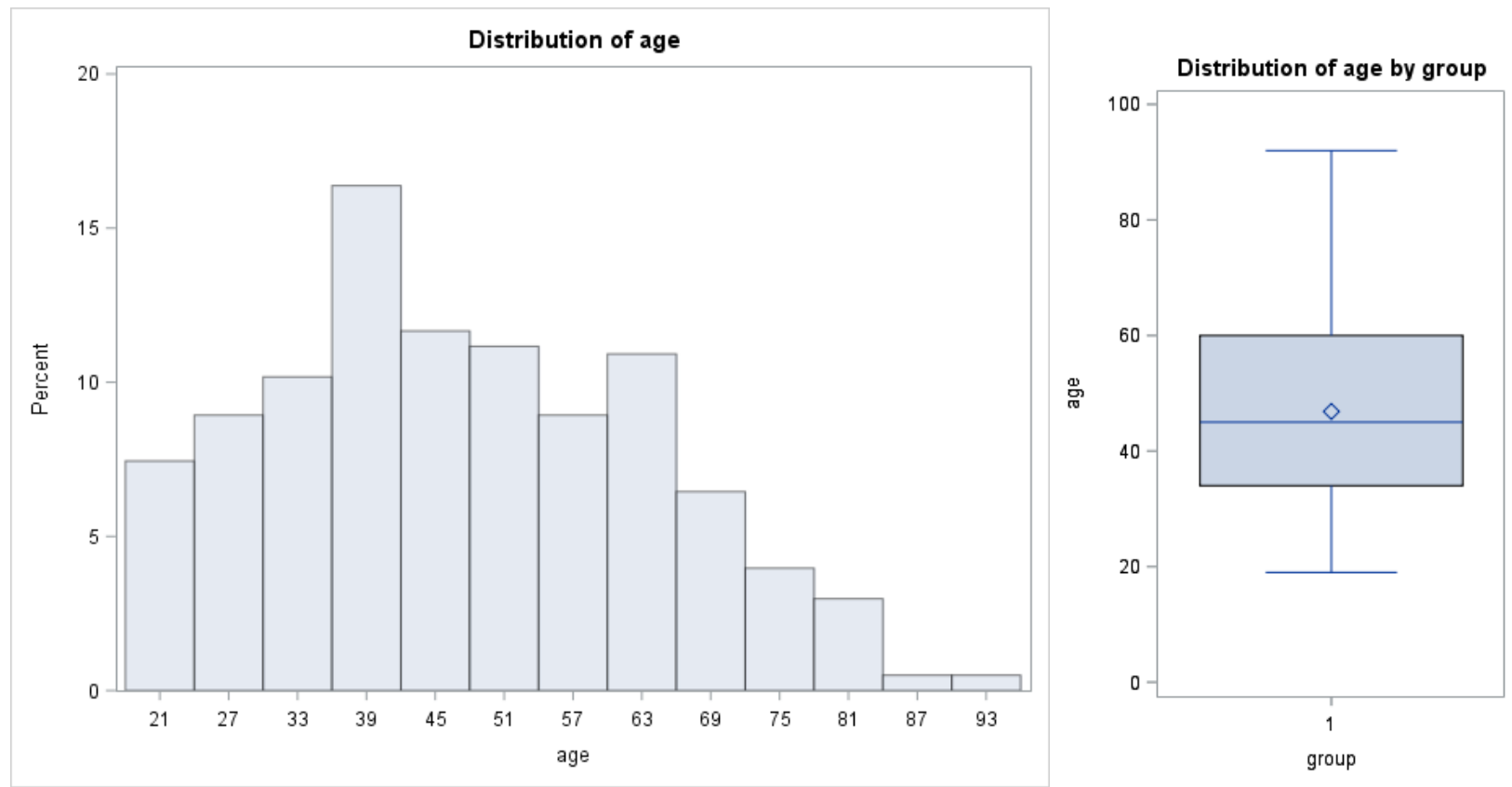

Figure appendix-5. The Histogram and Boxplot of the variable "age" is shown here.
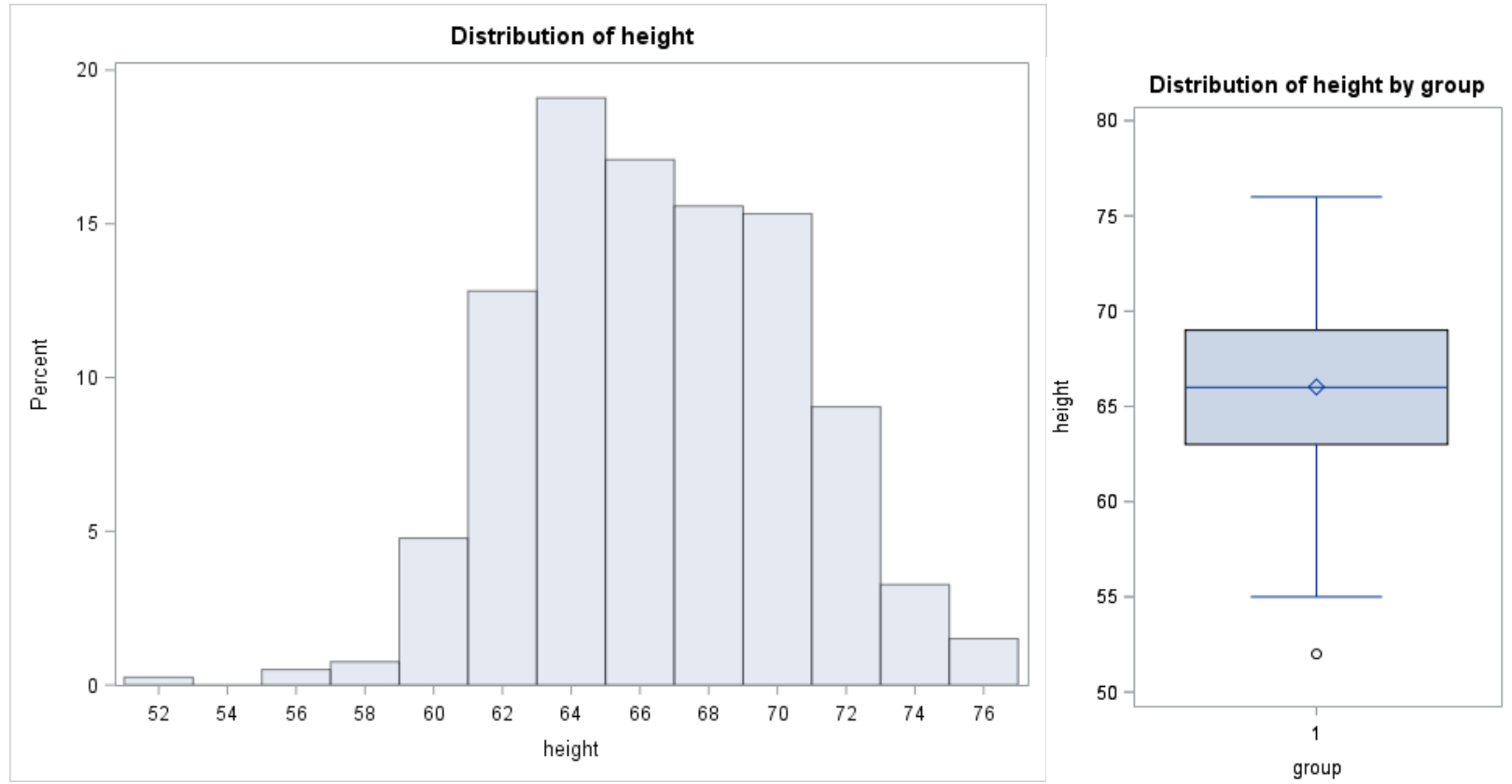

Figure appendix-6. The Histogram and Boxplot of the variable "height" is shown here. 

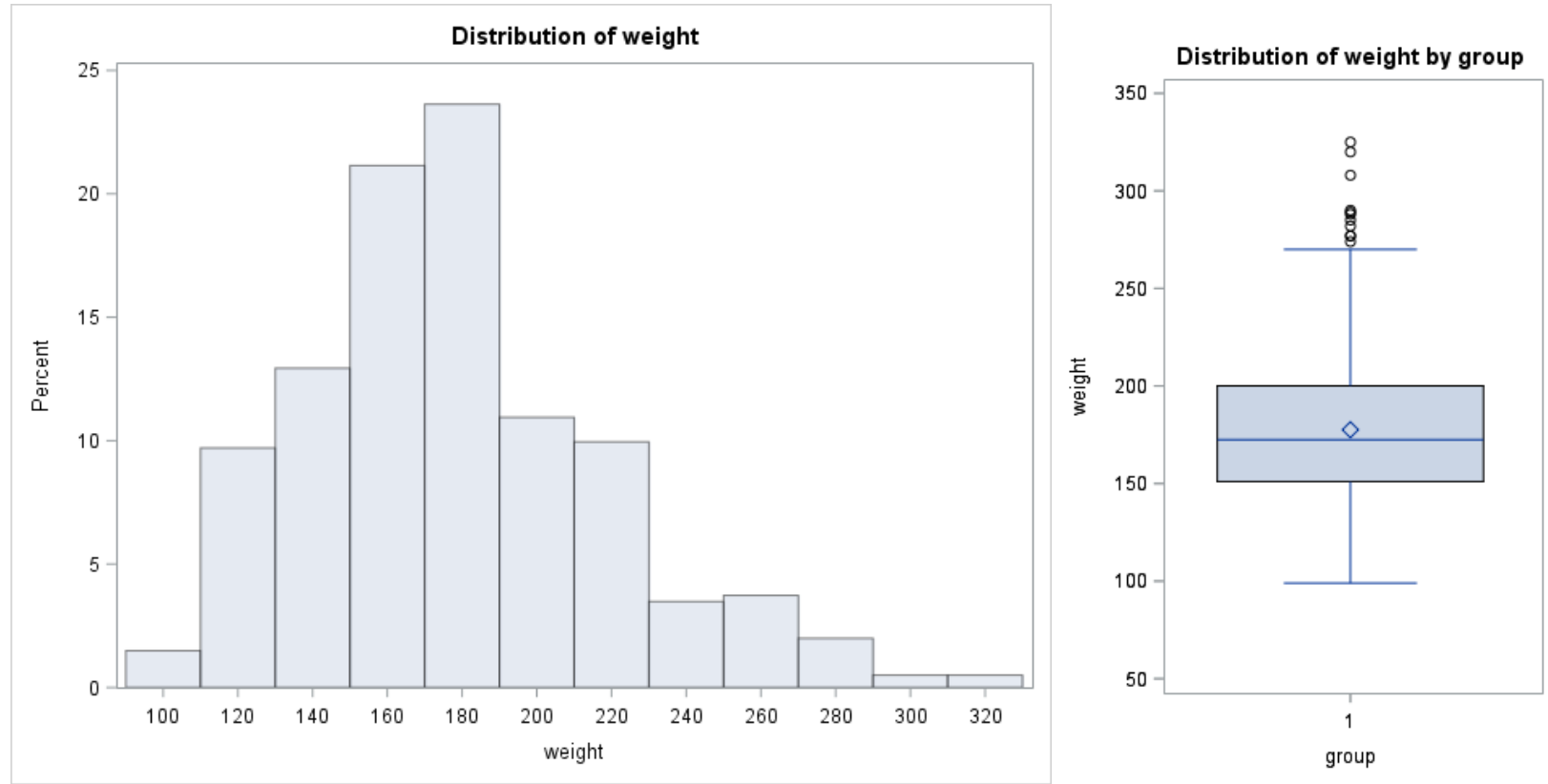

Figure appendix-7. The Histogram and Boxplot of the variable "weight" is shown here.
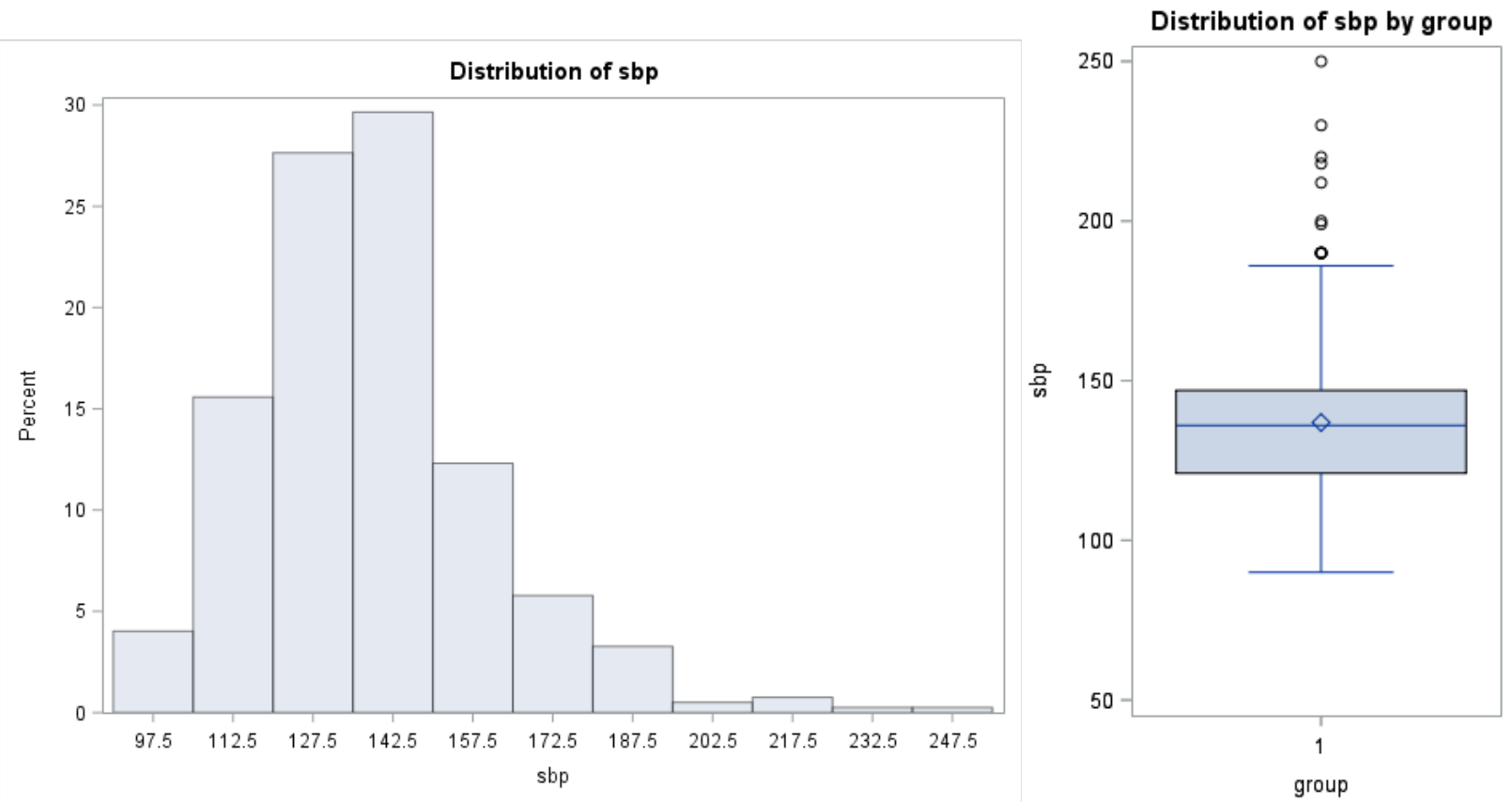

Figure appendix-8. The Histogram and Boxplot of the variable "sbp" is shown here. 

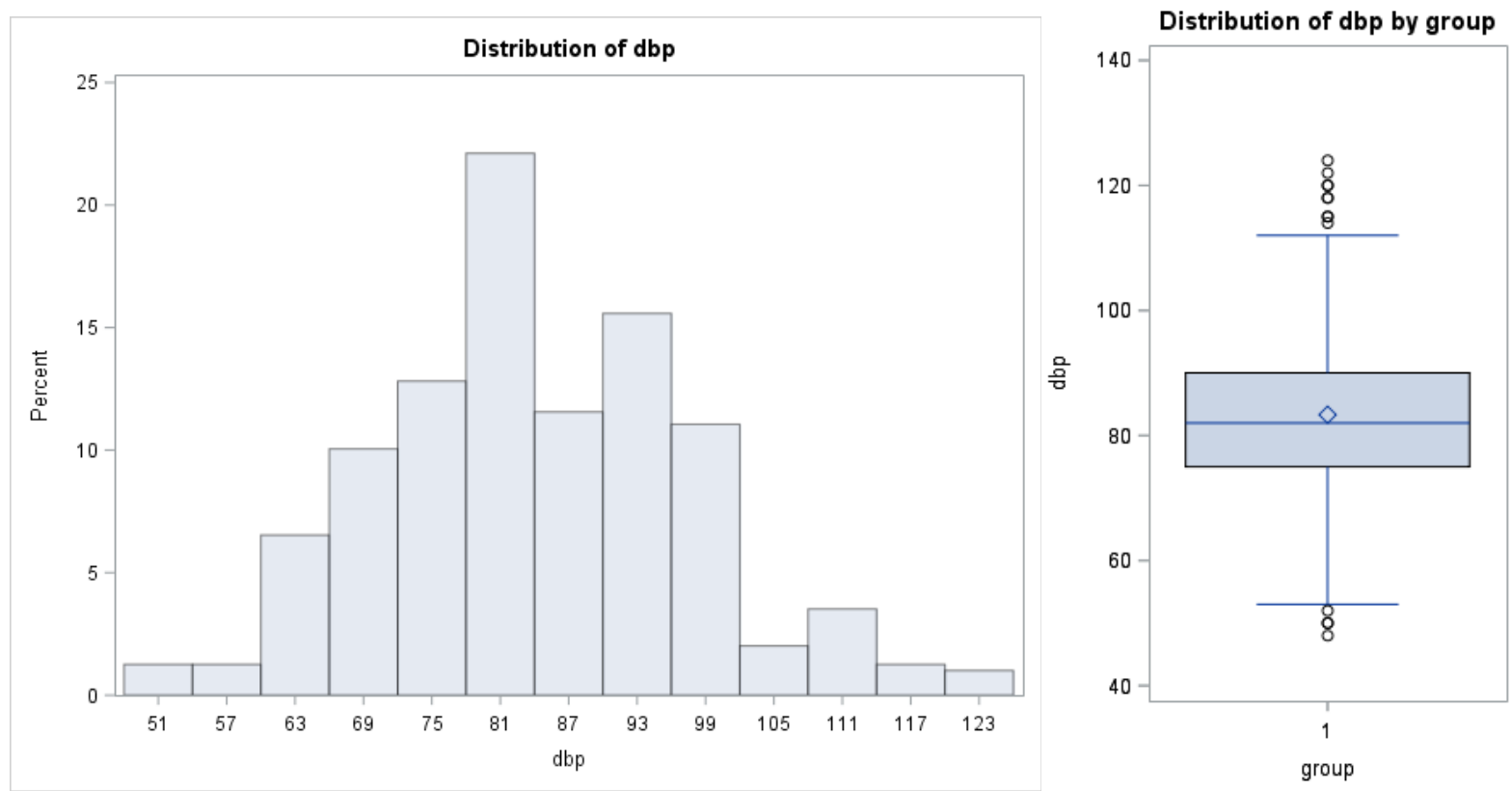

Figure appendix-9. The Histogram and Boxplot of the variable "dbp" is shown here.
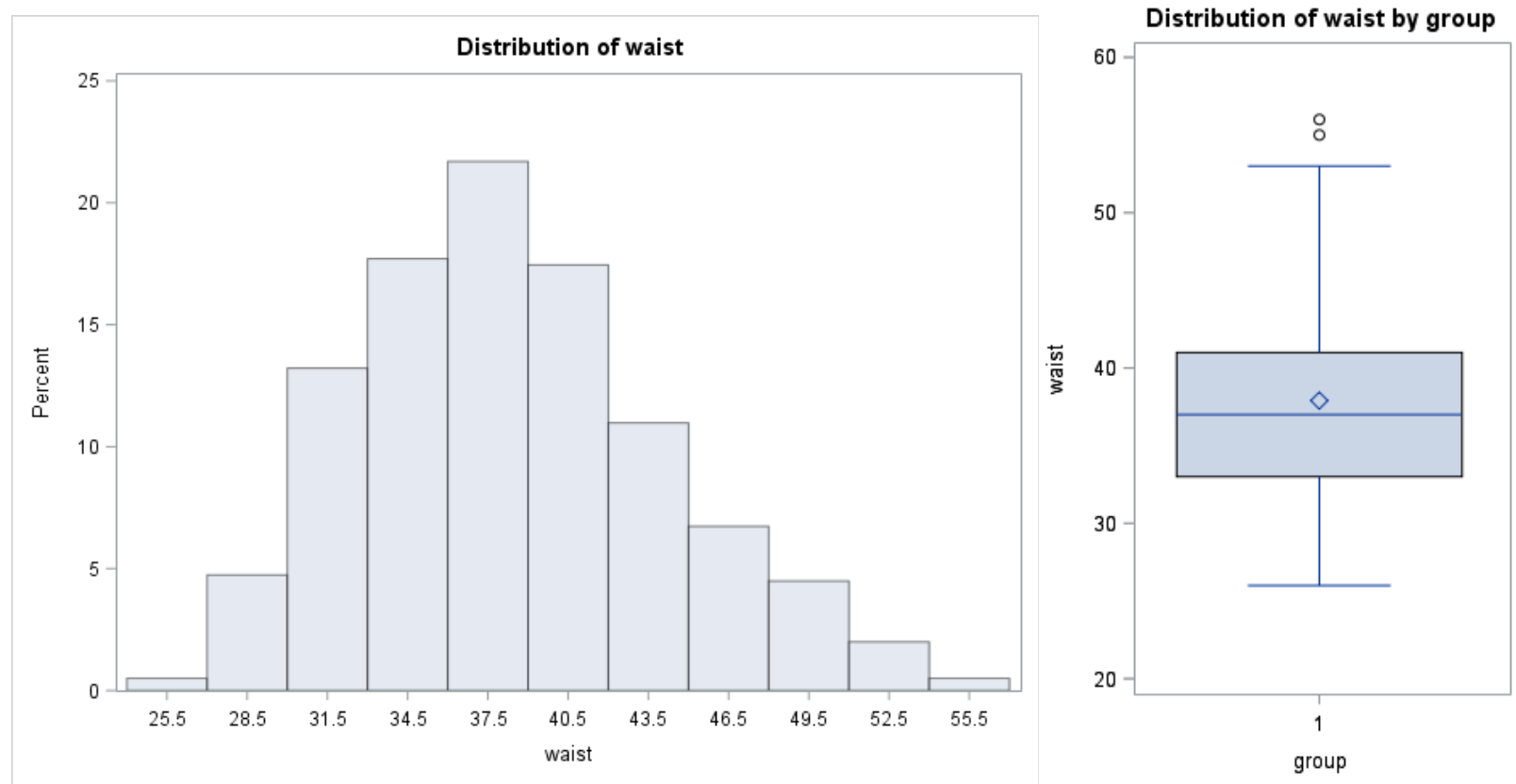

Figure appendix-10. The Histogram and Boxplot of the variable "waist" is shown here. 

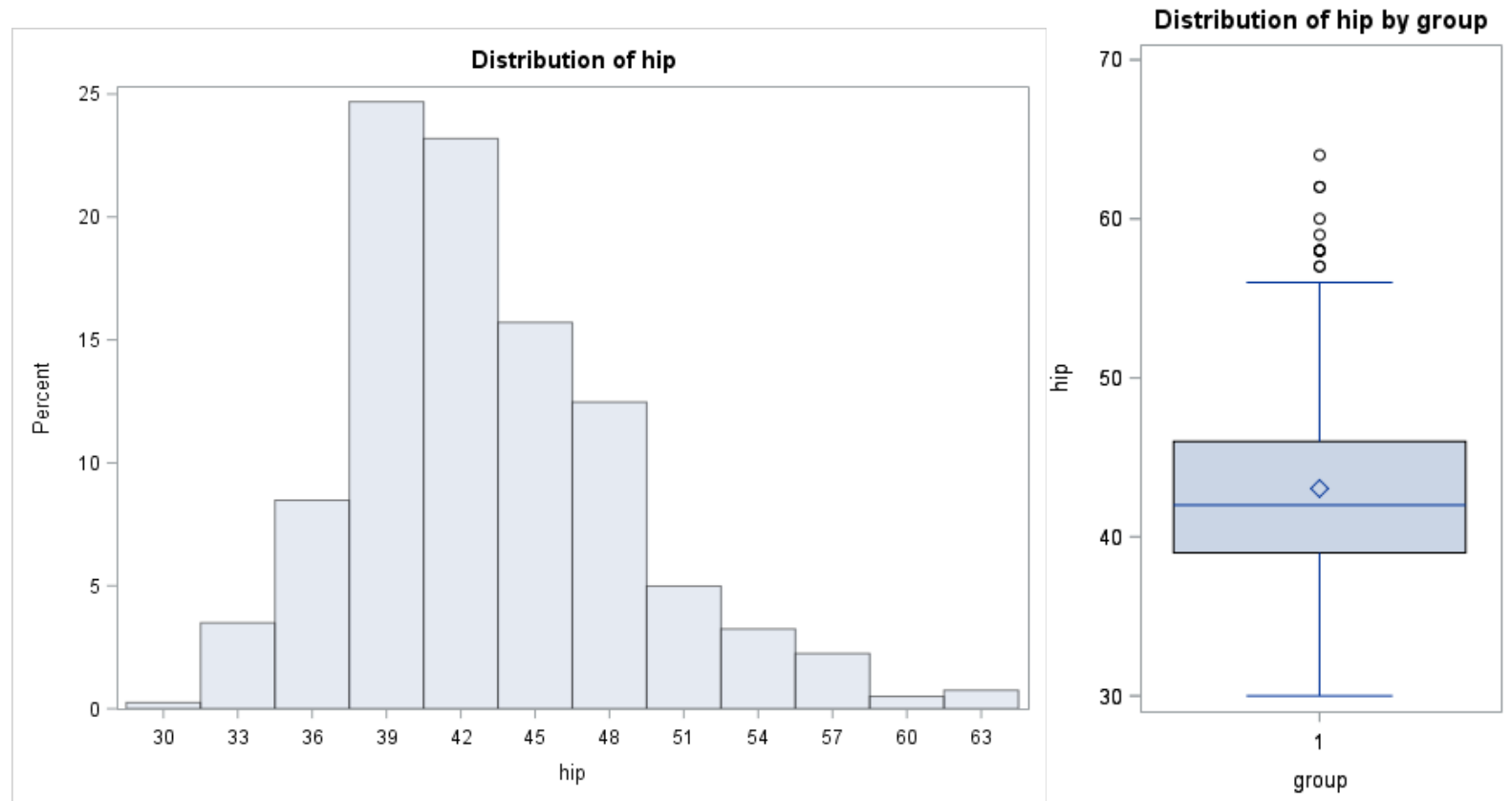

Figure appendix-11. The Histogram and Boxplot of the variable "hip" is shown here.
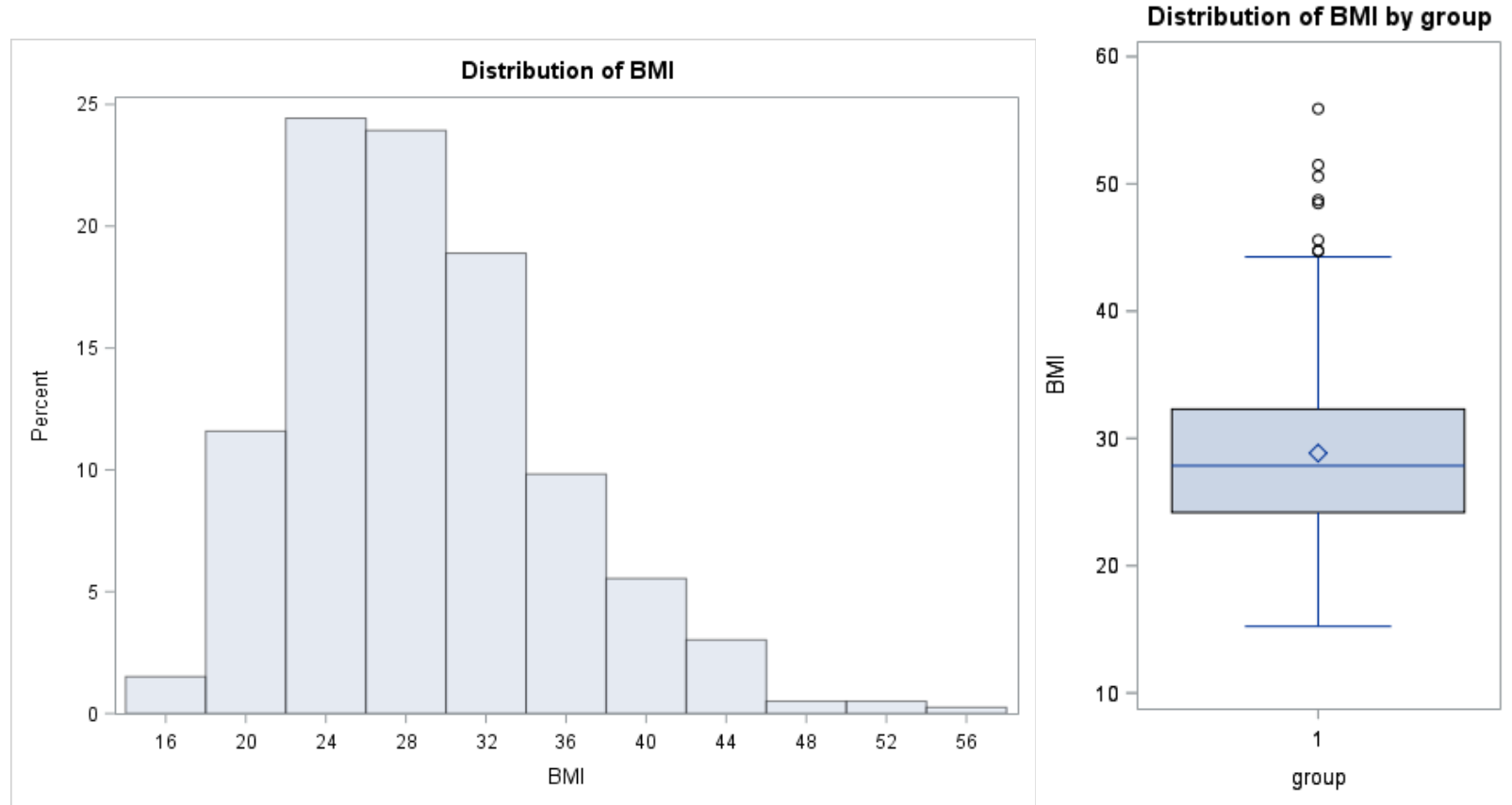

Figure appendix-12. The Histogram and Boxplot of the variable "BMI" is shown here. 
Table appendix-2. The p-value results of the Chi-square test of association

\begin{tabular}{l|l}
\hline VARIABLE & CHI-SQUARE P-VALUE \\
\hline WAIST & $<.0001$ \\
\hline HIP & $\mathbf{0 . 0 2 1 5}$ \\
BMI & $\mathbf{0 . 0 2 2 1}$ \\
AGE & $<.0001$ \\
SBP & $<.0001$ \\
CHOL & $\mathbf{0 . 0 0 2 3}$ \\
RATIO & $\mathbf{0 . 0 0 1 4}$ \\
WEIGHT & 0.0754 \\
HEIGHT & 0.6937 \\
DBP & 0.752 \\
HDL & 0.0717 \\
GENDER & 0.7591
\end{tabular}

This was not used to select the variables and the univariate analysis was used instead.

The goal of this test was to only give a bigger picture of the potential risk factors. 


\section{SAS Program}

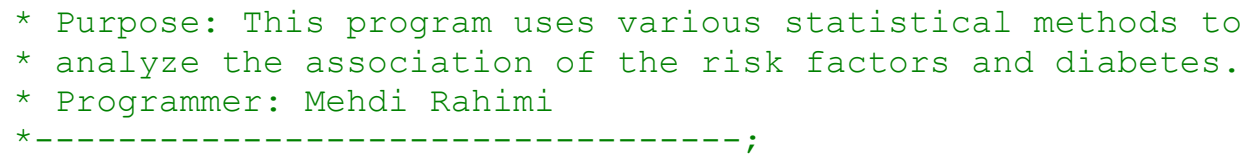




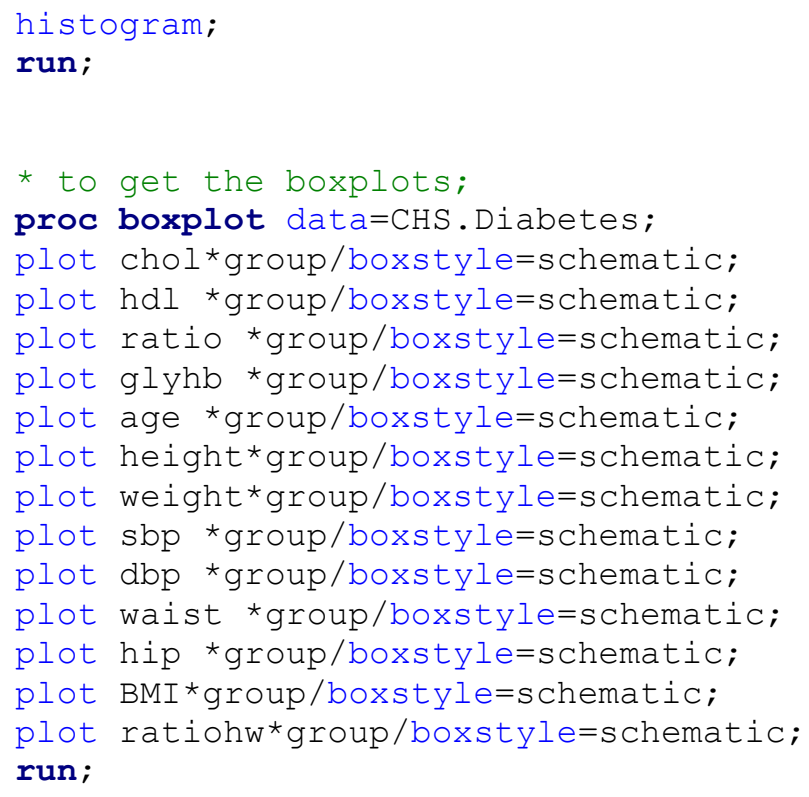

Page 25 of 28 


\begin{tabular}{|c|c|c|}
\hline & & $37-<\mathbf{4 1}=' 50$ th Pctl -75 th Pctl' \\
\hline & & $41-<60=' 75$ th Pctl $-\max ^{\prime} ;$ \\
\hline value & BMIg & $10-<24.18=' \min -25$ th Pctl' \\
\hline & & $24.18-<27.86=' 25$ th Pctl -50 th Pctl' \\
\hline & & $27.86-<32.31=' 50$ th Pctl -75 th Pctl' \\
\hline & & $32.31-<80=175$ th Pctl $-\max ^{\prime} ;$ \\
\hline value & hipg & $30-<39=' \min -25$ th Pctl' \\
\hline & & $39-<\mathbf{4 2}=$ '25th Pctl - 50th Pctl' \\
\hline & & $\mathbf{4 2 - < 4 6}=$ '50th Pctl -75 th Pctl' \\
\hline & & $46-<70=' 75$ th Pctl $-\max ^{\prime} ;$ \\
\hline value & weightG & $50-<151=' \min -25$ th Pctl' \\
\hline & & $151-<172.5=$ '25th Pctl - 50th Pctl' \\
\hline & & $172.5-<200=150$ th PCtl -75 th Pctl' \\
\hline & & $200-<400=175$ th $\operatorname{Pctl}-\max ' ;$ \\
\hline value & heightG & $50-<63=' \min -25$ th Pctl' \\
\hline & & $63-<66=$ '25th Pctl - 50th Pctl' \\
\hline & & $66-<69=' 50$ th Pctl -75 th Pctl' \\
\hline & & $69-<76=175$ th Pctl $-\max ^{\prime} ;$ \\
\hline value & ageG & $10-<34=' \mathrm{~min}-25 \mathrm{th}$ Pctl' \\
\hline & & $34-<45=$ '25th Pctl -50 th Pctl' \\
\hline & & $45-<60=' 50$ th Pctl -75 th Pctl' \\
\hline & & $60-<92=175$ th Pctl $-\max ^{\prime} ;$ \\
\hline value & sbpG & $80-<121=$ 'min -25 th Pctl' \\
\hline & & $121-<136=$ '25th Pctl -50 th Pctl' \\
\hline & & $136-<147=' 50$ th Pctl -75 th Pctl' \\
\hline & & $147-<260=' 75$ th Pctl $-\max ^{\prime} ;$ \\
\hline value & $\mathrm{dbpG}$ & $30-<75=$ 'min -25 th Pctl' \\
\hline & & $75-<82=' 25$ th Pctl -50 th Pctl' \\
\hline & & $82-<90=' 50$ th Pctl -75 th Pctl' \\
\hline & & $90-<130=' 75$ th Pctl $-\max ^{\prime} ;$ \\
\hline value & cholg & $75-<179=$ 'min -25 th Pctl' \\
\hline & & $\mathbf{1 7 9 - < 2 0 4}=$ '25th Pctl -50 th Pctl' \\
\hline & & $204-<230=' 50$ th Pctl - 75th Pctl' \\
\hline & & $230-<450=' 75$ th Pctl $-\max ^{\prime} ;$ \\
\hline value & hdlG & $10-<38=' \min -25$ th Pctl' \\
\hline & & $38-<46=' 25$ th Pctl -50 th Pctl' \\
\hline & & $46-<59=' 50$ th Pctl -75 th Pctl' \\
\hline & & $59-<130=175$ th Pctl $-\max ^{\prime} ;$ \\
\hline value & ratiog & $1-<3.2=' \mathrm{~min}-25 \mathrm{th}$ Pctl' \\
\hline & & $3.2-<4.2=' 25$ th Pctl -50 th Pctl' \\
\hline & & $4.2-<5.4=' 50$ th Pctl -75 th Pctl' \\
\hline & & $5.4-<20=175$ th $\operatorname{Pct} 1-\max ^{\prime} ;$ \\
\hline
\end{tabular}

run;

*HO: There is no association;

*HA: There is an association;

proc freq data=CHS.Diabetes;

*tables age/binomial;

tables disease*waist/chisq norow nocol nopercent;

tables disease*BMI/chisq norow nocol nopercent;

tables disease*hip/chisq norow nocol nopercent;

tables disease*weight/chisq norow nocol nopercent;

tables disease*height/chisq norow nocol nopercent;

tables disease*age/chisq norow nocol nopercent;

tables disease*sbp/chisq norow nocol nopercent;

tables disease*dbp/chisq norow nocol nopercent;

tables disease*chol/chisq norow nocol nopercent;

Page 26 of 28 
run; *gives log liklihood of -132.1470 ;

* model 2 - bmi2 - bmi3;

proc genmod data=CHS. Diabetes descending;

model disease = age ratio sbp/dist=bin link=logit;

run; *gives log liklihood of -138.2481;

* model 2 - sbp;

proc genmod data=CHS. Diabetes descending;

model disease = age ratio bmi2 bmi3/dist=bin link=logit;

run; *gives log liklihood of -133.0572;

* model 2 - ratio;

proc genmod data=CHS. Diabetes descending;

model disease = age sbp bmi2 bmi3/dist=bin link=logit;

run; *gives log liklihood of -140.7680 ;

* model 3 ;

proc genmod data=CHS. Diabetes descending;

model disease = age ratio bmi2 bmi3/dist=bin link=logit;

run; *gives log liklihood of -133.0572;

* model 3 - bmi2 - bmi3;

proc genmod data=CHS.Diabetes descending;

model disease = age ratio/dist=bin link=logit;

run; *gives log liklihood of -139.3227 ;

* model 3 - ratio;

proc genmod data $=$ CHS. Diabetes descending;

model disease = age bmi2 bmi3/dist=bin link=logit;

run; *gives log liklihood of -141.8020;

* test of OR;

proc genmod data=CHS. Diabetes descending;

model disease = age/dist=bin link=logit;

estimate 'Age OR' age 1/exp; * OR=1.0539;

run;

proc genmod data=CHS. Diabetes descending;

model disease $=$ bmi2 bmi3/dist=bin link=logit;

* 2 OR: ;

estimate 'Overweight vs. Normal' bmi2 1 bmi3 0/exp; * OR= 2.3322 and

significant;

estimate 'Obesity vs. Normal' bmi2 0 bmi3 1/exp; * OR=2.8702 and significant;

contrast 'Overall obesity' bmi2 1, bmi3 1; *overall oR:0.0188 for obesity

(although both were significant) ;

run; 